\title{
Choriocarcinoma syndrome after resection of primary pulmonary choriocarcinoma: report of a case
}

Tsuyoshi Takahashi ${ }^{1,2^{*}}$ (D) and Ryo Kobayashi ${ }^{1}$

\begin{abstract}
Background: Choriocarcinoma syndrome is known as a lethal complication from tumoral hemorrhage, which frequently occurs at the site of tumor metastasis.

Case presentation: A 59-year-old man with 60-pack-year smoking history was referred to our hospital because of hemoptysis. Chest computed tomography (CT) showed a $28 \times 18 \mathrm{~mm}$ spiculated nodule with a cavity infiltrating the left upper lobe. A transbronchial lung biopsy was performed, and histopathological examinations revealed adenocarcinoma. No distant or regional metastasis was observed, and therefore, the patient underwent a left upper lobectomy with lymphadenectomy. Histological examinations showed that the tumor consisted of poorly differentiated adenocarcinoma cells and a choriocarcinomatous component; no multiple pulmonary metastases and mediastinal lymph node metastasis were observed. Immunohistochemical analysis showed a positive immunoreaction for human chorionic gonadotropin in the syncytiotrophoblastic cells of the choriocarcinoma. One month after the operation, the patient developed massive hemoptysis. CT showed diffuse alveolar infiltration in both the lungs. A bronchoscopic examination showed bleeding from the right upper bronchus. Aspiration cytology showed carcinoma. Despite blood transfusion and management in the intensive care unit, the patient died one and a half month after diagnosis.

Conclusions: We herein report a case of a man who developed choriocarcinoma syndrome 1 month after resection for combined choriocarcinoma and adenocarcinoma of the lung. Choriocarcinoma syndrome is a rare and life-threatening complication which may occur in patients with primary pulmonary choriocarcinoma. However, we need to consider the risk of this syndrome while dealing with patients who have massive hemoptysis.
\end{abstract}

Keywords: Lung cancer, Choriocarcinoma, Choriocarcinoma syndrome, Lung cancer surgery

\section{Background}

Choriocarcinoma originates from anaplastic trophoblastic tissue and principally occurs in the female genital tract after a gestational event. Extragonadal choriocarcinoma is a rare entity; especially, primary pulmonary choriocarcinoma is an extremely rare entity. The lung is the most frequent site of metastasis from choriocarcinoma, and a lethal hemorrhagic comolication at the sites

\footnotetext{
* Correspondence: ttakahashi-tks@umin.ac.jp

'Department of Surgery, Yaizu City Hospital, 1000 Dobara, Yaizu City, Shizuoka 425-8505, Japan

${ }^{2}$ Present address: Department of Thoracic Surgery, Graduate School of Medicine, University of Tokyo, 7-3-1 Hongo, Bunkyo-ku, Tokyo 113-8655, Japan
}

of metastasis is well-recognized. This is named the choriocarcinoma syndrome, containing high-volume choriocarcinomatous elements with siginificant elevation of serum levels of beta-human chorionic gonadotropin (hCG) [1].

\section{Case presentation}

A 59-year-old man with a 60-pack-year smoking history was referred to our hospital due to hemoptysis. The patient had transurethral resection of the bladder tumor for bladder cancer. There were no abnormalities on physical examination, and laboratory data were within 
normal limits. His preoperative serum and urinary hCG levels were not examined. Chest computed tomography (CT) revealed a $28 \times 18 \mathrm{~mm}$ speculated cavitary nodule accompanied by parenchymal infiltration (Fig. 1). Positron emission tomography (PET)/CT showed a fluodeoxyglucose uptake in the nodule. Transbronchial lung biopsy revealed adenocarcinoma of the lung. In the absence of distant and regional metastasis, the patient was diagnosed as having stage IA [cT1bNOM0] lung adenocarcinoma and underwent a left upper lobectomy with lymphadenectomy. Histologic findings showed that the tumor was consisted of the choriocarcinomatous component (cytotrophoblastic and syncytiotriphoblastic cells) and poorly diffenrentiated adenocarcinoma (Fig. 2a). There were no multiple pulmonary metastasis and mediastinal lymph node metastasis. Immunohistochemistry showed a positive immunoreaction for hCG in the syncytiotrophoblastic cells of the choriocarcinoma (Fig. 2b).

The patient's postoperative course was uneventful. One month after the operation, the patient developed massive hemoptysis. CT revealed diffuse alveolar infiltrates in the both lungs (Fig. 3a). Bronchoscopic examination revealed massive bleeding from the right upper bronchus (Fig. 3b). Aspiration cytology from the right upper bronchus showed a choriocarcinoma component. Despite blood transfusion and management in the intensive care unit, the patient died.

\section{Discussion}

Choriocarcinoma is a germ cell tumor containing syncytiotrophoblastic cells and secreting hCG and most commonly occurs in the female genital tract after a gestational event such as hydatidiform mole, spontaneous abortion, ectopic pregnancy, or term pregnancy [2]. Primary pulmonary choriocarcinoma is an extremely rare entity and the pathogenesis of primary pulmonary choriocarcinoma has not been established yet. In our case, the tumor was consisted of a choriocarcinomatous component (cytotrophoblastic and syncytiotriphoblastic cells) and poorly diffenrentiated adenocarcinoma. To our knowledge, only one case of combined choriocarcinoma and adenocarcinoma of the lung has been reported by Chen et al. so far [3]. Chen et al. reported that there is the possibility that choriocarcinoma and adenocarcinoma arouse simultaneously or formed a collision tumor; however, it is considered that the choriocarcinoma might occur directly in the lung through trophoblastic differentiation of some cells in the former adenocarcinoma.

No established therapeutic guidelines are available for this clinical entity due to its rarity. In a small pulmonary choriocarcinoma, the first choice in the treatment may be surgical resection [4]. However, the natural course of choriocarcinoma is rapidly fatal in the great majority of cases, especially some patients presenting with choriocarcinoma syndrome which is characterized by life threatening bleeding at the sites of metastasis from choriocarcinoma may be threatened immediately by the complications of pulmonary hemorrhage. Hemoptysis, elevation of serum levels of hCG and the roentgenographic infiltrates with typical metastatic sharply defined nodules are characteristic with the choriocarcinoma syndrome [1]. In our case, unfortunately elevation of serum levels of hCG could not be confirmed because the patient suffered from massive hemoptysis and died before the pathological diagnosis of combined choriocarinoma and adenocarcinoma of the

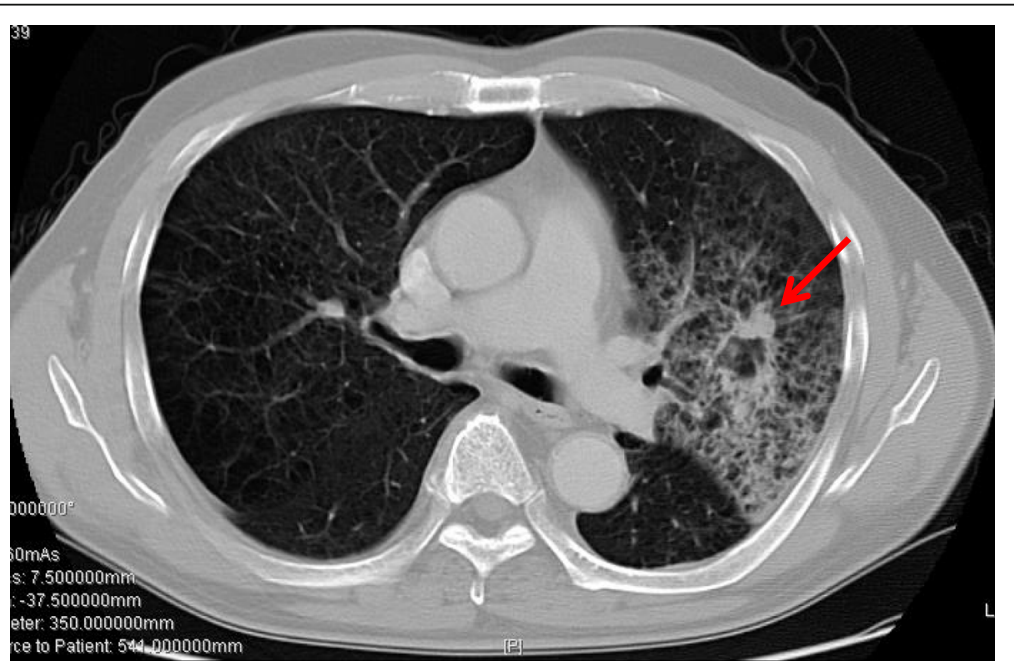

Fig. 1 Chest computed tomography (CT) revealed a $28 \times 18 \mathrm{~mm}$ spiculated cavitary nodule accompanied by parenchymal infiltration in the left upper lobe 


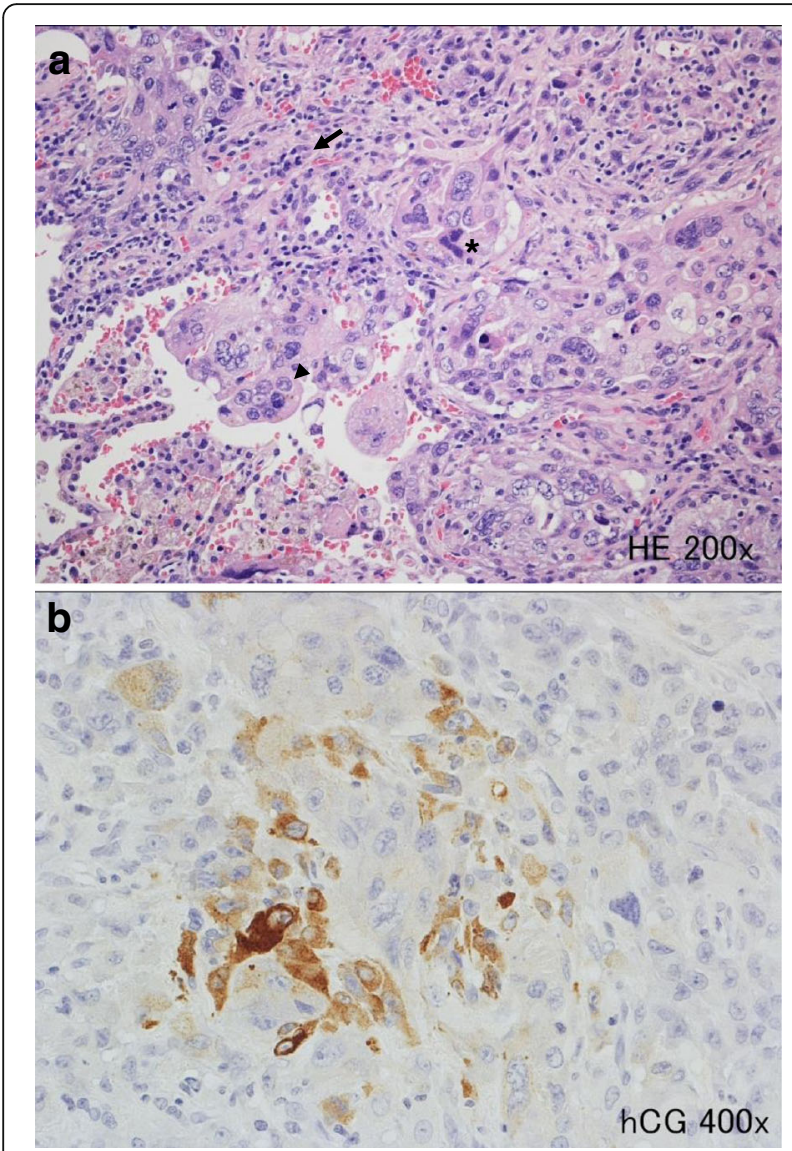

Fig. 2 a Histologic findings showing that the tumor was consisted of the poorly diffenrentiated adenocarcinoma (arrow) and a choriocarcinomatous component comprised of cytotrophoblastic (arrowhead) and syncytiotriphoblastic cells $\left(^{*}\right)$ (hematoxylin and eosin stain; original magnification, $\times 200$ ). b Immunohistochemistry showing a positive immunoreaction for beta-human chorionic gonadotropin (original magnification, $\times 400$ )

lung was made. Though chest CT revealed no pulmonary nodules suggestive of metastasis, we could make a definite diagnosis of choriocarcinoma syndrome from aspiration cytology. There is a report that combined modality therapy consisting of chemotherapy including bleomycin, etoposide, and cisplatin along with surgery and irradiation may result in a complete response [5]. Early recognition of choriocarcinoma syndrome and the urgent multimodality treatment consisted of surgery and chemotherapy might be beneficial.

\section{Conclusions}

Choriocarcinoma syndrome is a rare and life-threatening complication which may occur in patients with primary pulmonary choriocarcinoma. However, we need to consider the risk of this syndrome while dealing with patients who have massive hemoptysis.
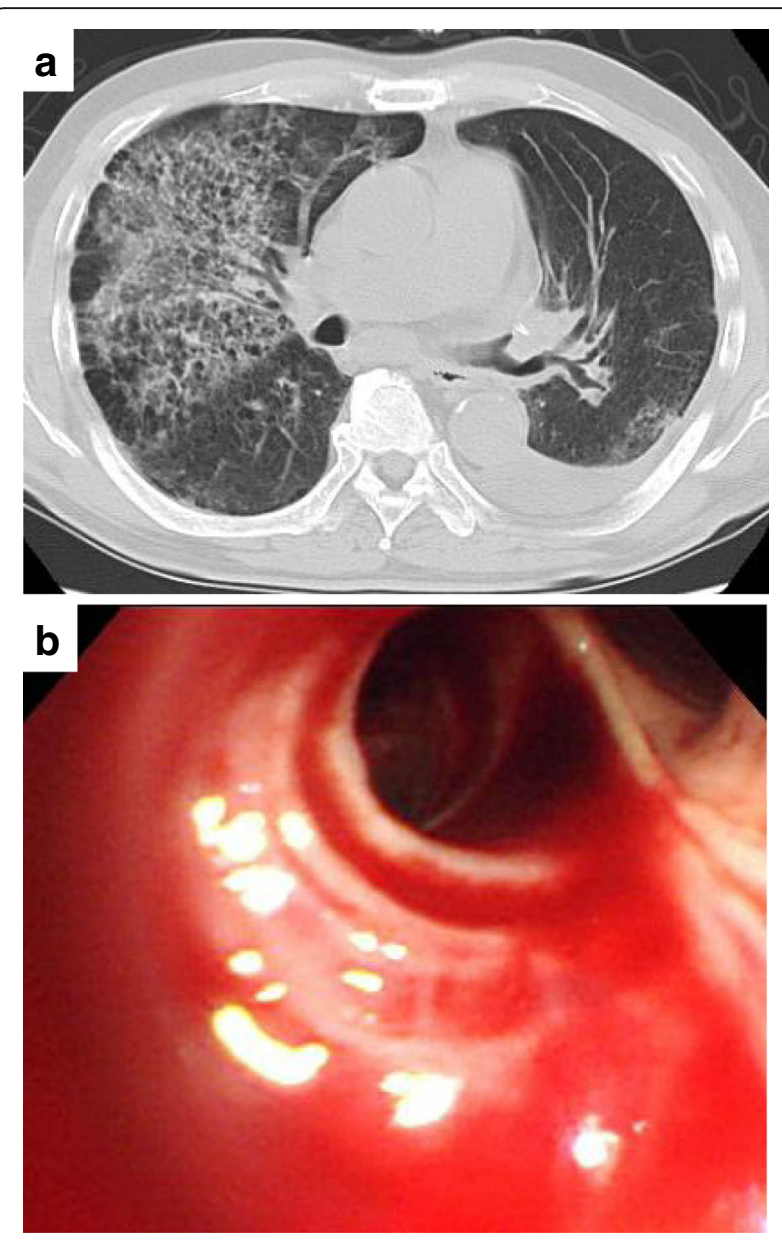

Fig. 3 a Chest computed tomography $(C T)$ revealed diffuse alveolar infiltrates in the both lungs. b Bronchoscopic examination revealed massive bleeding from the right upper bronchus

\section{Consent}

Written informed consent was obtained from the patient family for publication of this case report and any accompanying images. A copy of the written consent is available for review by the Editor-in-Chief of this journal.

\section{Abbreviations}

CT: Computed tomography; hCG: Human chorionic gonadotropin; PET: Positron emission tomography

\section{Acknowledgements}

None.

\section{Authors' contributions}

$\Pi T$ and RK participated in the collection, analysis, and interpretation of the data. $\Pi T$ wrote the draft manuscript. RK was revising the manuscript and study supervision. Both authors read and approved the final manuscript.

\section{Competing interests}

The authors declare that they have no competing interests.

Received: 12 August 2016 Accepted: 9 September 2016 Published online: 02 November 2016 


\section{References}

1. Logothetis CJ, Samuels ML, Selig DE, Oqden S, Dexeus F, Swanson D, et al. Cyclic chemotherapy with cyclophosphamide, doxorubicin and cisplatin plus vinblastine and bleomycin in advanced germinal tumors: results with 100 patients. Am J Med. 1986;81:219-28.

2. Venkatram S, Muppuri S, Niazi M, Fuentes GD. A 24-year-old pregnant patients with diffuse alveolar hemorrhage. Chest. 2010;138:220-3.

3. Chen F, Tatsumi A, Numoto S. Combined choriocarcinoma and adenocarcinoma of the lung occurring in a man. Cancer. 2001;91:123-9.

4. Canver CC, Voytovich MC. Resection of an unsuspected primary pulmonary choriocarcinoma. Ann Thorac Surg. 1996;61:1249-51.

5. Sridar KS, Saldana MJ, Thurer RJ, Beattie EJ. Primary choriocarcinoma of the lung: report of a case treated with intensive multimodality therapy and review of the literature. J Surg Oncol. 1989;41:93-7.

\section{Submit your manuscript to a SpringerOpen ${ }^{\circ}$ journal and benefit from:}

- Convenient online submission

- Rigorous peer review

- Immediate publication on acceptance

- Open access: articles freely available online

- High visibility within the field

- Retaining the copyright to your article

Submit your next manuscript at $\gg$ springeropen.com 